\title{
About sign-constancy of Green's function of a two-point problem for impulsive second order delay equations
}

\author{
Alexander Domoshnitsky ${ }^{\otimes 1}$, Guy Landsman ${ }^{2}$ and Shlomo Yanetz ${ }^{2}$ \\ ${ }^{1}$ Ariel University, Ariel, Israel \\ ${ }^{2}$ Bar Ilan University, Ramat Gan, Israel
}

Appeared 11 August 2016

Communicated by Tibor Krisztin

Abstract. We consider the following second order differential equation with delay

$$
\left\{\begin{array}{l}
(L x)(t) \equiv x^{\prime \prime}(t)+\sum_{j=1}^{p} a_{j}(t) x^{\prime}\left(t-\tau_{j}(t)\right)+\sum_{j=1}^{p} b_{j}(t) x\left(t-\theta_{j}(t)\right)=f(t), \quad t \in[0, \omega] \\
x\left(t_{k}\right)=\gamma_{k} x\left(t_{k}-0\right), x^{\prime}\left(t_{k}\right)=\delta_{k} x^{\prime}\left(t_{k}-0\right), \quad k=1,2, \ldots, r .
\end{array}\right.
$$

In this paper we find sufficient conditions of positivity of Green's functions for this impulsive equation coupled with two-point boundary conditions in the form of theorems about differential inequalities.

Choosing the test function in these theorems, we obtain simple sufficient conditions.

Keywords: impulsive equations, Green's functions, positivity/negativity of Green's functions, boundary value problem, second order.

2010 Mathematics Subject Classification: 34K10, 34B37, 34A40, 34A37, 34K48.

\section{Introduction}

Let us consider the following impulsive equations:

$$
\begin{gathered}
(L x)(t) \equiv x^{\prime \prime}(t)+\sum_{j=1}^{p} a_{j}(t) x^{\prime}\left(t-\tau_{j}(t)\right)+\sum_{j=1}^{p} b_{j}(t) x\left(t-\theta_{j}(t)\right)=f(t), \quad t \in[0, \omega] \\
x\left(t_{k}\right)=\gamma_{k} x\left(t_{k}-0\right), \quad x^{\prime}\left(t_{k}\right)=\delta_{k} x^{\prime}\left(t_{k}-0\right), \quad k=1,2, \ldots, r \\
0=t_{0}<t_{1}<t_{2}<\cdots<t_{r}<t_{r+1}=\omega,
\end{gathered}
$$

\footnotetext{
${ }^{\bowtie}$ Corresponding author. Email: adom@ariel.ac.il

This paper is part of the second author's Ph.D. thesis which is being carried out in the Department of Mathematics at Bar-Ilan University.
} 


$$
x(\zeta)=0, \quad \zeta<0
$$

where $f, a_{j}, b_{j}:[0, \omega] \rightarrow \mathbb{R}$ are summable functions and $\tau_{j}, \theta_{j}:[0, \omega] \rightarrow[0,+\infty)$ are measurable functions for $j=1,2, \ldots, p$. Here $p$ and $r$ are natural numbers, $\gamma_{k}$ and $\delta_{k}$ are real positive numbers.

Let $D$ be a space of functions $x:[0, \omega] \rightarrow \mathbb{R}$ such that their derivative $x^{\prime}(t)$ is absolutely continuous on every interval $t \in\left[t_{i}, t_{i+1}\right), i=0, \ldots, r, x^{\prime \prime} \in L_{\infty}$, there exist the finite limits $x\left(t_{i}-0\right)=\lim _{t \rightarrow t_{i}^{-}} x(t)$ and $x^{\prime}\left(t_{i}-0\right)=\lim _{t \rightarrow t_{i}^{-}} x^{\prime}(t)$ and condition (1.2) is satisfied at points $t_{i}(i=0, \ldots, r)$. We understand solution $x$ as a function $x \in D$ satisfying (1.1)-(1.3). For equation (1.1) we consider the following variants of boundary conditions:

$$
\begin{array}{rlrl}
x(0) & =\alpha_{0}, & x^{\prime}(0) & =\beta_{0}, \\
x(0) & =\alpha_{0}, & x^{\prime}(\omega) & =\beta_{0}, \\
x^{\prime}(0) & =\alpha_{0}, \quad x(\omega) & =\beta_{0} .
\end{array}
$$

Differential equations with impulses have attracted the attention of many researchers. Note the monographs $[2,4,18,22,23,26]$, in which problems of existence, uniqueness and stability are considered.

In the works $[7,15,18,19,22,23,26]$, impulsive ordinary differential equations are considered. Let us assume that all trajectories of solutions to non-impulsive ordinary differential equation are known. In this case, impulses imply only choosing the trajectory between the points of impulses, but we stay on trajectories of corresponding solutions of a non-impulsive equation between the points of impulses $t_{i}$ and $t_{i+1}$. In the case of impulsive equation with delay it is not true anymore. That is why properties of delay impulsive equations can be quite different. Oscillation/nonoscillation and stability of delay differential equations are considered in $[1,5,6,8,9,25]$. Delay impulsive differential equations of second order are considered concerning stabilization by impulses in $[14,20]$. For second order delay differential equations, we note the paper [24] where their nonoscillation is studied. There are almost no results about boundary value problems for impulsive differential equations of high orders. Note that second order ordinary impulsive differential equations are considered in $[3,7,15]$. The Dirichlet boundary value problem is studied in [21] and the generalized Dirichlet problem in [13,16,21]. For delay differential equations, there is only the paper [10].

Let us introduce a function $C(t, s): C(\cdot, s)$, as a function of $t$, for every fixed $s: t_{i_{s}}<s<$ $t_{i_{s}+1}\left(i_{s}=0, \ldots, r\right)$, satisfies the equation

$$
\begin{gathered}
x^{\prime \prime}(t)+\sum_{j=1}^{p} a_{j}(t) x^{\prime}\left(t-\tau_{j}(t)\right)+\sum_{j=1}^{p} b_{j}(t) x\left(t-\theta_{j}(t)\right)=0, \quad s \leq t, \\
x\left(t_{k}\right)=\gamma_{k} x\left(t_{k}-0\right), \quad x^{\prime}\left(t_{k}\right)=\delta_{k} x^{\prime}\left(t_{k}-0\right), \quad k=i_{s}+1, \ldots, r, \\
t_{i_{s}}<s<t_{i_{s}+1}<\cdots<t_{r}<t_{r+1}=\omega, \\
x(\zeta)=0, \quad \zeta<s .
\end{gathered}
$$

and the initial conditions $C(s, s)=0, \frac{\partial}{\partial t} C(s, s)=1$. Note that $C(t, s)=0$ for $t<s$. It is clear that for every $s$ this function is defined uniquely. We call this function $C(t, s)$ as the Cauchy function of (1.1)-(1.3). From the formula of solutions' representation for system of delay 
impulsive equations (see [9]) follows that the general solution of (1.1)-(1.3) can be represented in the form

$$
x(t)=v_{1}(t) x(0)+v_{2}(t) x^{\prime}(0)+\int_{0}^{t} C(t, s) f(s) d s
$$

where $v_{1}, v_{2}$ are the solutions of the homogeneous equation (1.12), (1.2), (1.3) where

$$
(L x)(t)=0, \quad t \in[0, \omega]
$$

satisfying the initial conditions

$$
v_{1}(0)=1, \quad v_{1}^{\prime}(0)=0, \quad v_{2}(0)=0, \quad v_{2}^{\prime}(0)=1 .
$$

According to the definition of $C(t, s)$ it is clear that $C(t, 0)=v_{2}(t)$.

Note for example, that for the auxiliary equation (1.14), (1.2), (1.3) where

$$
x^{\prime \prime}(t)=f(t),
$$

we obtained in [11] the following formula for its Cauchy function $C_{0}(t, s)$

$$
\begin{aligned}
C_{0}(t, s)=\sum_{i=1}^{r} \sum_{j=0}^{i-1}\left[\prod_{k=j+1}^{i} \gamma_{k}\left(t_{j+1}-s\right)+\sum_{l=j+2}^{i} \prod_{k=l}^{i} \gamma_{k} \prod_{k=j+1}^{l-1} \delta_{k}\left(t_{l}-t_{l-1}\right)+\prod_{k=j+1}^{i} \delta_{k}\left(t-t_{i}\right)\right] \\
\quad \times\left[H_{t_{i}}(t)-H_{t_{i+1}}(t)\right]\left[H_{t_{j}}(s)-H_{t_{j+1}}(s)\right] \\
+\sum_{i=0}^{r} H_{S}(t)(t-s)\left[H_{t_{i}}(t)-H_{t_{i+1}}(t)\right]\left[H_{t_{i}}(s)-H_{t_{i+1}}(s)\right]
\end{aligned}
$$

where $H_{t_{i}}(t)$ is the Heaviside function

$$
H_{t_{i}}(t)= \begin{cases}1, & t_{i} \leq t \\ 0, & t<t_{i} .\end{cases}
$$

From the general theory of functional differential equations [2] we have the following fact. If every one of the boundary value problems, of equation (1.12) with a corresponding condition

$$
\begin{aligned}
x(0) & =0, & x^{\prime}(0) & =0 \\
x(0) & =0, & x^{\prime}(\omega) & =0 \\
x^{\prime}(0) & =0, & x(\omega) & =0
\end{aligned}
$$

has only trivial solutions, then their solution can be represented in the form

$$
x(t)=\int_{0}^{t} G(t, s) f(s) d s,
$$

where $G(t, s)$ is called the Green's function of the corresponding problem. The form of Green's function $G(t, s)$ of every problem can be obtained using the representation (1.11) of general solution of (1.1)-(1.3).

In this paper, we develop the approach of [9] for second order impulsive equations (1.1)(1.3). This approach is based on the construction of Green's functions of auxiliary impulsive equations. Note that for first order functional differential equations, these Green's functions 
even for nonlocal boundary value problems are constructed in [12]. We construct Green's functions for two auxiliary boundary value problems for second order impulsive equations. Our approach is based on a reduction of the impulsive boundary value problem to an integral equation; and then corresponding Krasnoselskii's theorems about estimates of the spectral radius are used [17]. On this basis, we obtain theorems on differential inequalities allowing to make the conclusion about sign constancy of Green's functions. Choosing the test functions, we get conditions of positivity/negativity of the Green's functions.

Our paper is constructed as follows. After introducing the main questions in the introduction, we construct Green's function of the auxiliary problems in Section 2. In Section 3, we demonstrate graphs of Green's functions of the axuiliary problems. Then we discuss negativity of these Green's functions and their derivatives in Section 4. In Section 5, we obtain the main results of the paper in the form of assertions about differential and integral inequalities. Efficient tests are also obtained on this basis in Section 5.

\section{About Green's functions for the auxiliary boundary value problem}

We want to obtain a representation of the Green's function $G_{0}^{1}(t, s)$ of the auxiliary boundary value problem (1.14), (1.2), (1.3), (1.5). We use the second boundary condition $x^{\prime}(\omega)=\beta_{0}$ in order to find a representation of $x^{\prime}(0)$ through $\alpha_{0}$ and $\beta_{0}$. From the general solution (1.11) of equation (1.1)-(1.3), we get

$$
x^{\prime}(\omega)=\beta_{0}=v_{1}^{\prime}(\omega) \alpha_{0}+\left[\frac{\partial}{\partial t} C_{0}(t, 0)\right]_{t=\omega} x^{\prime}(0)+\sum_{j=1}^{r} \int_{t_{j}}^{t_{j+1}}\left[\frac{\partial}{\partial t} C_{0}(t, s)\right]_{t=\omega} f(s) d s .
$$

In [11] it was obtained that

$$
v_{1}(t)=\prod_{i=1}^{r} \gamma_{i}, \quad t \in\left[t_{r}, \omega\right]
$$

and $v_{1}^{\prime}(\omega)=0$. From here, we obtain for problem (1.14), (1.2), (1.3), (1.5) that

$$
x^{\prime}(0)=\frac{\beta_{0}-\sum_{j=1}^{r} \frac{\partial}{\partial t} \int_{t_{j}}^{t_{j+1}}\left[C_{0}(t, s)\right]_{t=\omega} f(s) d s}{\left[\frac{\partial}{\partial t} C_{0}(t, 0)\right]_{t=\omega}}
$$

where

$$
\frac{\partial}{\partial t} C_{0}(t, s)= \begin{cases}\frac{\partial}{\partial t} C_{0}(t, s), & t \neq t_{k} \\ \delta_{k} \frac{\partial}{\partial t} C_{0}\left(t_{k}-0, s\right), & t=t_{k} .\end{cases}
$$

where $\delta_{k}$ defines the impulse of the derivative at the point $t_{k}$. The general solution for the auxiliary boundary value problem with (1.5) can be represented now in the form:

$$
\begin{aligned}
x(t)= & \prod_{i=1}^{j} \gamma_{i} \alpha_{0}+C_{0}(t, 0) \frac{\beta_{0}}{\left[\frac{\partial}{\partial t} C_{0}(t, 0)\right]_{t=\omega}} \\
& +\int_{0}^{\omega}\left[C_{0}(t, s)-C_{0}(t, 0) \frac{\left[\frac{\partial}{\partial t} C_{0}(t, s)\right]_{t=\omega}}{\left[\frac{\partial}{\partial t} C_{0}(t, 0)\right]_{t=\omega}}\right] f(s) d s, \quad t \in\left(t_{j}, t_{j+1}\right) .
\end{aligned}
$$


Thus the Green's function $G_{0}^{1}(t, s)$ of problem (1.14), (1.2), (1.3), (1.5) is

$$
G_{0}^{1}(t, s)=C_{0}(t, s)-C_{0}(t, 0) \frac{\left[\frac{\partial}{\partial t} C_{0}(t, s)\right]_{t=\omega}}{\left[\frac{\partial}{\partial t} C_{0}(t, 0)\right]_{t=\omega}}, \quad t \in\left(t_{j}, t_{j+1}\right)
$$

Summarizing, we have obtained the actual representation of $G_{0}^{1}(t, s)$ and formulate the following lemma.

Lemma 2.1. The general solution for the auxiliary boundary value problem with impulses (1.14), (1.2), (1.3), (1.5) can be represented in the form:

$$
x(t)=V_{1}(t)+\int_{0}^{\omega} G_{0}^{1}(t, s) f(s) d s, \quad t \in[0, \omega]
$$

where the Green's function $G_{0}^{1}(t, s)$ of this problem is

$$
G_{0}^{1}(t, s)=C_{0}(t, s)-C_{0}(t, 0) \frac{\left[\frac{\partial}{\partial t} C_{0}(t, s)\right]_{t=\omega}}{\left[\frac{\partial}{\partial t} C_{0}(t, 0)\right]_{t=\omega}} \quad t \in\left(t_{j}, t_{j+1}\right)
$$

where the Cauchy function $C_{0}(t, s)$ of this problem is defined by (1.15) with $C_{0}(t, s)=0$ for $t<s$ and

$$
V_{1}(t)=\prod_{i=1}^{j} \gamma_{i} \alpha_{0}+C_{0}(t, 0) \frac{\beta_{0}}{\left[\frac{\partial}{\partial t} C_{0}(t, 0)\right]_{t=\omega}}, \quad t \in\left[t_{j}, t_{j+1}\right), \quad j=0,1, \ldots, r, \quad t_{0}=0 .
$$

Let us describe a formula for $G_{0}^{1}(t, s)$ using the formula of $C_{0}(t, s)$ described by (1.15), $t \in\left[t_{i}, t_{i+1}\right), s \in\left[t_{j}, t_{j+1}\right)$. We get

$$
G_{0}^{1}(t, s)=C_{i j}(t, s)-C_{i 0}(t, 0) \frac{C_{r j}(\omega, s)\left(\Delta_{t_{r}}(\omega)-\Delta_{t_{r+1}}(\omega)\right)+\prod_{k=j+1}^{r} \delta_{k}}{C_{r 0}(\omega, 0)\left(\Delta_{t_{r}}(\omega)-\Delta_{t_{r+1}}(\omega)\right)+\prod_{k=1}^{r} \delta_{k}}
$$

where

$$
C_{i j}(t, s)= \begin{cases}\prod_{k=j+1}^{i} \gamma_{k}\left(t_{j+1}-s\right)+\sum_{l=j+2}^{i} \prod_{k=l}^{i} \gamma_{k} \prod_{k=j+1}^{l-1} \delta_{k}\left(t_{l}-t_{l-1}\right) & \\ \quad+\prod_{k=j+1}^{i} \delta_{k}\left(t-t_{i}\right) & i>j \\ t-s, & i=j, \\ 0, & i<j,\end{cases}
$$

and $\Delta_{t_{r}}$ is the Dirac delta function.

Let us get a representation of the Green's function $G_{2}(t, s)$ of a general second order linear differential equation with (1.6). The general solution for this problem is presented in equation (1.11). Let us use the second boundary condition $x(\omega)=\beta_{0}$ in order to find a representation of $x(0)$ through $\alpha_{0}$ and $\beta_{0}$. From the general solution of the problem, we get

$$
\beta_{0}=x(\omega)=x(0) v_{1}(\omega)+x^{\prime}(0) v_{2}(\omega)+\int_{0}^{\omega} C(\omega, s) f(s) d s .
$$

From here, we obtain

$$
x(0)=-\frac{1}{v_{1}(\omega)} \int_{0}^{\omega} C(\omega, s) f(s) d s-\alpha_{0} \frac{v_{2}(\omega)}{v_{1}(\omega)}+\beta_{0} \frac{1}{v_{1}(\omega)}
$$


and the general solution can be represented in the form:

$$
x(t)=-\alpha_{0} v_{1}(t) \frac{v_{2}(\omega)}{v_{1}(\omega)}+\alpha_{0} v_{2}(t)+\beta_{0} v_{1}(t) \frac{1}{v_{1}(\omega)}+\int_{0}^{\omega}\left[C(t, s)-\frac{v_{1}(t)}{v_{1}(\omega)} C(\omega, s)\right] f(s) d s .
$$

Thus the Green's function $G_{2}(t, s)$ of this problem is

$$
G_{2}(t, s)=C(t, s)-\frac{v_{1}(t)}{v_{1}(\omega)} C(\omega, s) .
$$

For our specific case, we have $v_{1}(t)=\prod_{i=1}^{j} \gamma_{i}, t \in\left[t_{j}, t_{j+1}\right), v_{2}(t)=C_{0}(t, 0)$ and $C(t, s)=$ $C_{0}(t, s)$. Substituting $v_{1}(t), v_{2}(t)$ and $C(t, s)$ into this formula, we obtain the following lemma for problem (1.14), (1.2), (1.3), (1.6).

Lemma 2.2. The general solution for the auxiliary boundary value problem with impulses (1.14), (1.2), (1.3), (1.6) can be represented in the form:

$$
x(t)=V_{2}(t)+\int_{0}^{\omega} G_{0}^{2}(t, s) f(s) d s, \quad t \in[0, \omega],
$$

where the Green's function $G_{0}^{2}(t, s)$ of this problem is

$$
G_{0}^{2}(t, s)=C_{0}(t, s)-\frac{\prod_{i=1}^{j} \gamma_{i}}{\prod_{i=1}^{r} \gamma_{i}} C_{0}(\omega, s), \quad t \in\left[t_{j}, t_{j+1}\right),
$$

where the Cauchy function $C_{0}(t, s)$ of this problem defined by $(1.15)$ with $C_{0}(t, s)=0$ for $t<s$ and

$$
V_{2}(t)=-\alpha_{0} C_{0}(\omega, 0) \frac{\prod_{i=1}^{j} \gamma_{i}}{\prod_{i=1}^{r} \gamma_{i}}+\alpha_{0} C_{0}(t, 0)+\beta_{0} \frac{\prod_{i=1}^{j} \gamma_{i}}{\prod_{i=1}^{r} \gamma_{i}}, \quad t \in\left[t_{j}, t_{j+1}\right) .
$$

Let us construct a formula for $G_{0}^{2}(t, s)$ in another form. By using the formula of $C_{0}(t, s)$ we obtain that for $t \in\left[t_{i}, t_{i+1}\right), s \in\left[t_{j}, t_{j+1}\right)$

$$
G_{0}^{2}(t, s)=C_{i j}(t, s)-C_{r j}(\omega, s) \frac{\prod_{k=1}^{j} \gamma_{k}}{\prod_{k=1}^{r} \gamma_{k}} .
$$

\section{Graphs of Green's functions for auxiliary problems}

Let us construct the graph of the Green's function of (1.14), (1.2), (1.3), (1.5). According to the properties of Green's function (see [2]), the Green's function $G_{0}^{1}(t, s)$ of the problem (1.14), (1.2), (1.3), (1.5) is a solution of the equation $x^{\prime \prime}(t)=0$. We obtain Figure 3.1 in the case $r=4$.

Let us construct the graph of the Green's function of (1.14), (1.2), (1.3), (1.6). According to the properties of Green's function (see [2]), the Green's function $G_{0}^{2}(t, s)$ of the problem (1.14), $(1.2),(1.3),(1.6)$ is a solution of the equation $x^{\prime \prime}(t)=0$. We obtain Figure 3.2 in the case $r=4$.

\section{Sign constancy of the Green's functions and their derivatives for the auxiliary impulsive equation}

In this section, we prove positivity or negativity of the derivatives of Green's function for one and two-point impulsive problems with the auxiliary equation $x^{\prime \prime}(t)=f(t)$. 


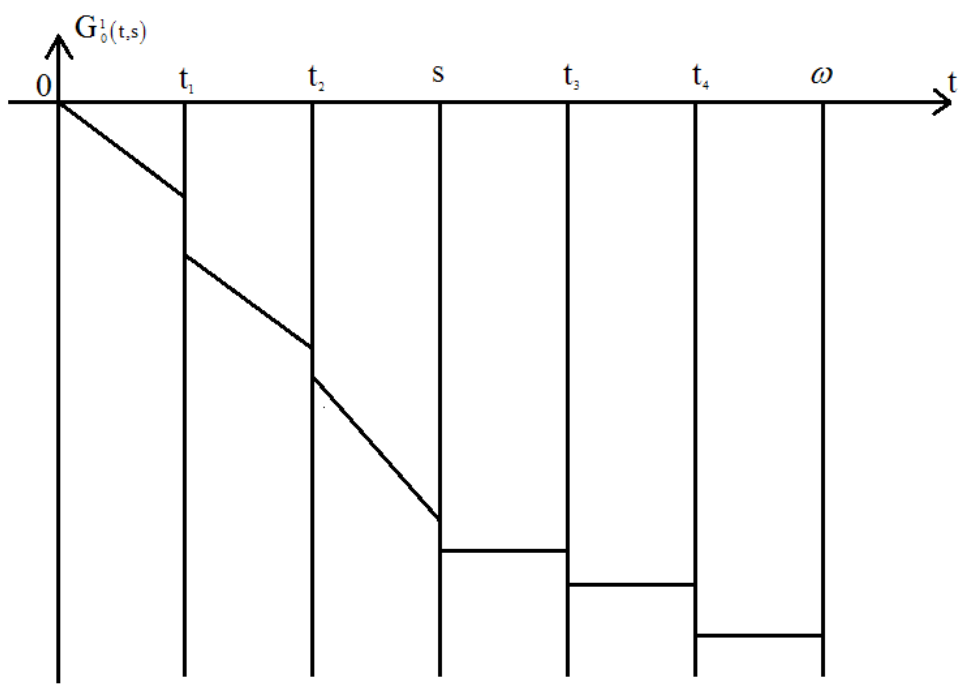

Figure 3.1: $G_{0}^{1}(t, s)$ for $s \in(0, \omega)$.

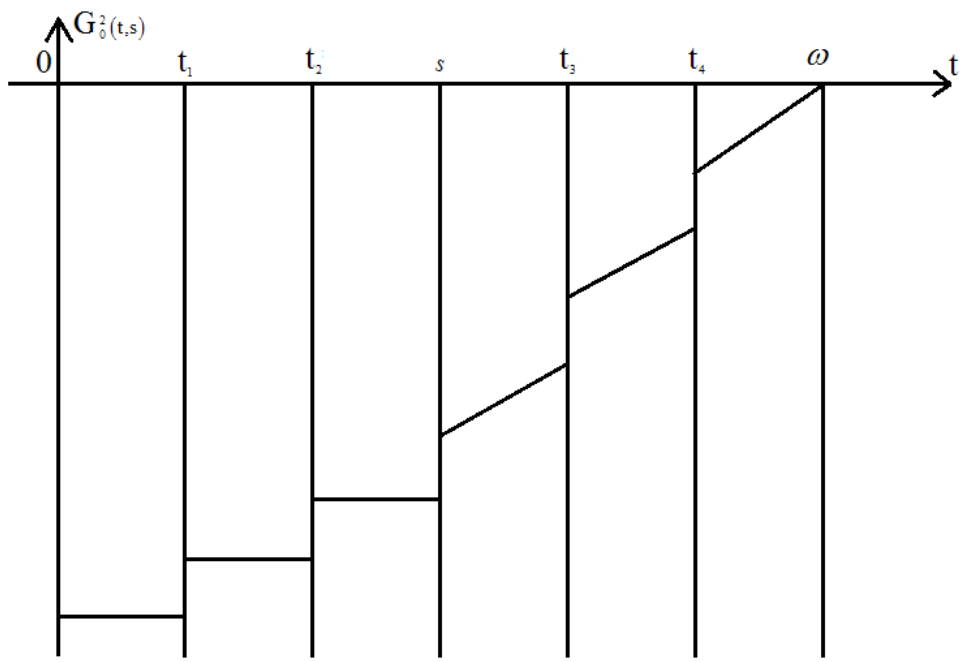

Figure 3.2: $G_{0}^{2}(t, s)$ for $s \in(0, \omega)$.

Lemma 4.1. If $1 \leq \gamma_{k}, 1 \leq \delta_{k}, k=1, \ldots, r$, then the operators $C_{0}: L_{\infty} \rightarrow L_{\infty}$ and $C_{0}^{\prime}: L_{\infty} \rightarrow L_{\infty}$ defined by

$$
\begin{aligned}
& \left(C_{0} f\right)(t)=\int_{0}^{t} C_{0}(t, s) f(s) d s \\
& \left(C_{0}^{\prime} f\right)(t)=\frac{d}{d t} \int_{0}^{t} C_{0}(t, s) f(s) d s
\end{aligned}
$$

are positive. 
Proof. It is clear from (1.15) (see [11]) that $C_{0}$ is a positive operator. Let us assume that $t \in$ $\left(t_{i}, t_{i+1}\right)$ and $s \in\left(t_{j}, t_{j+1}\right)$, where $i \geq j, i, j=0, \ldots, r, t_{0}=0$. If $i=j$, then $\frac{\partial}{\partial t} C_{0}(t, s)=1 \geq 0$. If $i>j$, then

$$
\frac{\partial}{\partial t} C_{0}(t, s)=\prod_{k=j+1}^{i} \delta_{k} \geq 0,
$$

since $\delta_{k}>0, k=1, \ldots, r$. Since $1 \leq \gamma_{k}, 1 \leq \delta_{k}$ it is clearly to see that the operator $C_{0}^{\prime}$ is also positive. Lemma 4.1 has been proven.

Lemma 4.2. If $1 \leq \gamma_{k}, 1 \leq \delta_{k}, k=1, \ldots, r$, then the operators $G_{0}^{1}: L_{\infty} \rightarrow L_{\infty}$ and $G_{0}^{1^{\prime}}: L_{\infty} \rightarrow L_{\infty}$ defined by

$$
\begin{aligned}
\left(G_{0}^{1} f\right)(t) & =\int_{0}^{\omega} G_{0}^{1}(t, s) f(s) d s \\
\left(G_{0}^{1^{\prime}} f\right)(t) & =\frac{d}{d t} \int_{0}^{\omega} G_{0}^{1}(t, s) f(s) d s
\end{aligned}
$$

are negative.

Proof. Let us assume that $t \in\left(t_{i}, t_{i+1}\right)$ and $s \in\left(t_{j}, t_{j+1}\right)$, where $i<j, i, j=0, \ldots, r, t_{0}=0$. Then

$$
\frac{\partial}{\partial t} G_{0}^{1}(t, s)=-\frac{\partial}{\partial t} C_{0}(t, 0) \frac{\left[\frac{\partial}{\partial t} C_{0}(t, s)\right]_{t=\omega}}{\left[\frac{\partial}{\partial t} C_{0}(t, 0)\right]_{t=\omega}}=-\prod_{k=1}^{i} \delta_{k} \frac{\prod_{k=j+1}^{r} \delta_{k}}{\prod_{k=1}^{r} \delta_{k}}<0 .
$$

For $i>j$, it is clear that $\frac{\partial}{\partial t} G_{0}^{1}(t, s)=0$. For $i=j$ we get $\frac{\partial}{\partial t} G_{0}^{1}(t, s)<0$ if $t<s$ and $\frac{\partial}{\partial t} G_{0}^{1}(t, s)=0$ if $s>t$. From here, it is clear that the operator $G_{0}^{1^{\prime}}$ is negative. Since $1 \leq \gamma_{k}, 1 \leq \delta_{k}$, it is clear that the operator $G_{0}^{1}$ negative (see for example Figure 3.1). Lemma 4.2 has been proven.

Let us consider the following example demonstrating that this operator defined by (4.4) in the case $0<\gamma_{1}<1$ is not negative.

The function

$$
x(t)=\left\{\begin{array}{l}
\frac{t^{2}}{2}+2 t, \quad t \in[0,2), \\
\frac{t^{2}}{2}-4 t+6 \gamma+6, \quad t \in[2,4],
\end{array}\right.
$$

is a solution of the problem

$$
\left\{\begin{array}{l}
x^{\prime \prime}(t)=1, \quad t \in[0,4] \\
x(0)=0, x^{\prime}(4)=0 \\
x(2)=\gamma x(2-0), x^{\prime}(2)=x^{\prime}(2-0)
\end{array}\right.
$$

It can be easily observed that the function $x(t)$ monotone on $[0,4]$ if and only if $\gamma \geq 1$.

Lemma 4.3. If $0<\gamma_{k} \leq 1,0<\delta_{k} \leq 1, k=1, \ldots, r$, then the operator $G_{0}^{2}: L_{\infty} \rightarrow L_{\infty}$ defined by

$$
\left(G_{0}^{2} f\right)(t)=\int_{0}^{\omega} G_{0}^{2}(t, s) f(s) d s
$$

is negative and the operator $G_{0}^{2^{\prime}}: L_{\infty} \rightarrow L_{\infty}$ defined by

$$
\left(G_{0}^{2^{\prime}} f\right)(z)=\frac{d}{d t} \int_{0}^{\omega} G_{0}^{2}(t, s) f(s) d s
$$

is positive. 
Proof. From the formula of $G_{0}^{2}(t, s)$ it is clear that $\frac{\partial}{\partial t} G_{0}^{2}(t, s)=\frac{\partial}{\partial t} C_{0}(t, s)$ for every $t \neq t_{i}$ and for almost every $s$. Hence, the operators $C_{0}^{\prime}$, defined by (4.2) and $G_{0}^{2^{\prime}}$ defined by (4.6) coincide. From Lemma 4.1 the operator $C_{0}^{\prime}$ is positive, so the operator $G_{0}^{2^{\prime}}$ is also positive. Since $0<\gamma_{k} \leq 1,0<\delta_{k} \leq 1$, it is clear that the operator $G_{0}^{2}$ is negative (see for example Figure 3.2). Lemma 4.3 has been proven.

\section{Nonpositivity of Green's function for the two-point impulsive problems}

In this section, we prove theorems about the negativity of the Green's functions $G_{1}(t, s)$ and $G_{2}(t, s)$ for the given problems (1.1)-(1.3), (1.5) and (1.1)-(1.3), (1.6). Then we will demonstrate examples in order to find sufficient conditions for their negativity.

Theorem 5.1. Assume that $a_{j} \geq 0, b_{j} \geq 0$ for $j=1, \ldots, p, 1 \leq \gamma_{k}, 1 \leq \delta_{k}$, for $k=1, \ldots, r$ and there exists the function $v \in D$ and $\epsilon>0$ such that

$$
(L v)(t) \leq-\epsilon<0, \quad v(t)>0, \quad v^{\prime}(t)>0, \quad v^{\prime \prime}(t)<0, \quad t \in(0, \omega),
$$

where the differential operator $L$ is defined by (1.1). Then the Green's function $G_{1}(t, s)$ of $(1.1)-(1.3)$, (1.5) satisfies the inequality $G_{1}(t, s) \leq 0,(t, s) \in[0, \omega] \times[0, \omega]$.

Proof. Let $v^{\prime \prime}(t)=z(t)$ where $z \in L_{\infty}$, then we can write

$$
v(t)=\int_{0}^{\omega} G_{0}^{1}(t, s) z(s) d s+V_{1}(t)
$$

where $G_{0}^{1}(t, s)$ is the Green's function of the problem (1.14), (1.2), (1.3), (1.5). After substitution we obtain

$$
z(t)=\left(K_{1} z\right)(t)+(L v)(t)-\sum_{j=1}^{p} a_{j}(t) V_{1}^{\prime}\left(t-\tau_{j}(t)\right)-\sum_{j=1}^{p} b_{j}(t) V_{1}\left(t-\theta_{j}(t)\right),
$$

where

$$
\begin{aligned}
\left(K_{1} z\right)(t)= & -\sum_{j=1}^{p} a_{j}(t) \int_{0}^{\omega} \frac{\partial}{\partial t} G_{0}^{1}\left(t-\tau_{j}(t), s\right) z(s) d s \\
& -\sum_{j=1}^{p} b_{j}(t) \int_{0}^{\omega} G_{0}^{1}\left(t-\theta_{j}(t), s\right) z(s) d s
\end{aligned}
$$

where $\frac{\partial}{\partial t} G_{0}^{1}\left(t-\tau_{j}(t), s\right)=0$ if $t-\tau_{j}(t)<0$ and $G_{0}^{1}\left(t-\theta_{j}(t), s\right)=0$ if $t-\theta_{j}(t)<0$. By Lemma 4.2, in the case $1 \leq \gamma_{k}, 1 \leq \delta_{k}$, we have positivity of the operator $K_{1}: L_{\infty} \rightarrow L_{\infty}$. Now, from the condition about existence of $v$ satisfying (5.1), we get that $z(t)=v^{\prime \prime}(t)<$ $0, \tilde{z}(t)=-z(t)>0$ and then there exists $\epsilon>0$ such that $\tilde{z}(t)-\left(K_{1} \tilde{z}\right)(t)=-(L v)(t)+$ $\sum_{j=1}^{p} a_{j}(t) V_{1}^{\prime}\left(t-\tau_{j}(t)\right)+\sum_{j=1}^{p} b_{j}(t) V_{1}\left(t-\theta_{j}(t)\right) \geq \epsilon$. We can write the assertion obtained in $[17$, p. 86] for our case in the following form.

Lemma 5.2. If there exists a function $z \in L_{\infty}$ and positive $\epsilon$ such that $z(t) \geq \epsilon, z(t)-\left(K_{1} z\right)(t) \geq \epsilon$ for $t \in(0, \omega)$, then $\rho\left(K_{1}\right)<1$. 
From here, the spectral radius $\rho\left(K_{1}\right)$ of the operator $K_{1}: L_{\infty} \rightarrow L_{\infty}$ is less than one.

Assume now that $f \geq 0$ and demonstrate that $x \leq 0$. After the substitution

$$
x(t)=\int_{0}^{\omega} G_{0}^{1}(t, s) z(s) d s+V_{1}(t)
$$

we obtain

$$
z(t)=\left(K_{1} z\right)(t)+f(t)-\sum_{j=1}^{p} a_{j}(t) V_{1}^{\prime}\left(t-\tau_{j}(t)\right)-\sum_{j=1}^{p} b_{j}(t) V_{1}\left(t-\theta_{j}(t)\right),
$$

where $K_{1}$ is positive operator and its spectral radius $\rho\left(K_{1}\right)<1$. Then

$$
z(t)=\left(I-K_{1}\right)^{-1} f(t)=f(t)+K_{1} f(t)+K_{1}^{2} f(t)+\cdots
$$

is nonnegative, and $x$ obtained by (5.5) is nonpositive. Since this is true for every nonnegative function $f$, we can make a conclusion that the solution of problem (1.1)-(1.3), (1.5) exists for every summable function $f$. Now it is clear that the solution of this problem has the integral representation (see (1.20)) with the kernel $G_{1}(t, s)$ (Green's function of (1.1)-(1.3), (1.5)). We proved that, for every nonpositive right hand side function $f$, the solution $x$ is nonpositive. From here, it follows that $G_{1}(t, s) \leq 0$.

Theorem 5.1 has been proven.

Example 5.3. Let us now find an example of a function $v$ satisfying the condition of Theorem 5.1. To this end, let us start with $v(t)=t(2 \omega-t)$ in the interval $t \in\left[0, t_{1}\right)$ where $\epsilon$ is a small positive constant. The function $v$ in the rest of the intervals will be of the form

$$
v(t)=v\left(t_{i}\right)+v^{\prime}\left(t_{i}\right)\left(t-t_{i}\right)-\left(t-t_{i}\right)^{2}, \quad t \in\left[t_{i}, t_{i+1}\right), \quad i=1, \ldots, r, \quad t_{r+1}=\omega
$$

where

$$
\left\{\begin{array}{l}
v\left(t_{i}\right)=\gamma_{i} v\left(t_{i}-0\right) \\
v^{\prime}\left(t_{i}\right)=\delta_{i} v^{\prime}\left(t_{i}-0\right)
\end{array}\right.
$$

Thus

$$
\left\{\begin{array}{l}
v(t)=t(2 \omega-t), \quad t \in\left[0, t_{1}\right), \\
v(t)=v\left(t_{i}\right)+v^{\prime}\left(t_{i}\right)\left(t-t_{i}\right)-\left(t-t_{i}\right)^{2}, \quad t \in\left[t_{i}, t_{i+1}\right),
\end{array}\right.
$$

where $v\left(t_{i}\right)$ and $v^{\prime}\left(t_{i}\right)$ can be presented in the forms

$$
\left\{\begin{aligned}
v\left(t_{i}\right)= & t_{1}\left(2 \omega-t_{1}\right) \prod_{j=1}^{i} \gamma_{j}+\sum_{k=2}^{i} v^{\prime}\left(t_{k}\right)\left(t_{k}-t_{k-1}\right) \prod_{j=k}^{i} \gamma_{j} \\
& -\sum_{k=2}^{i}\left(t_{k}-t_{k-1}\right)^{2} \prod_{j=k}^{i} \gamma_{j}, \\
v^{\prime}\left(t_{i}\right)= & 2\left(\omega-t_{1}\right) \prod_{j=1}^{i} \delta_{j}-2 \sum_{k=2}^{i}\left(t_{k}-t_{k-1}\right) \prod_{j=k}^{i} \delta_{j} .
\end{aligned}\right.
$$

Let us assume that $v(t)>0$ and substitute this $v(t)$ into condition (5.1) of Theorem 5.1.

For the next corollary, we use the following notation:

$$
\begin{aligned}
& \Omega_{1}=\max _{i=1,2, \ldots, r}\left[v^{\prime}\left(t_{i}\right)-2 t_{i}\right], \\
& \Omega_{2}=\max \left[\max _{i=1,2, \ldots, r} v\left(\frac{v^{\prime}\left(t_{i}\right)}{2}+t_{i}\right), \max _{i=0,1, \ldots, r} v\left(t_{i}\right)\right],
\end{aligned}
$$

where $v\left(t_{r+1}\right)=v(\omega)$. 
Corollary 5.4. If $a_{j} \geq 0, b_{j} \geq 0,1 \leq \gamma_{k}, 1 \leq \delta_{k}, j=1, \ldots, p, v(t)$ defined by (5.10) is positive for $t \in(0, \omega)$ and

$$
\Omega_{1} \sum_{j=1}^{p} a_{j}(t)+\Omega_{2} \sum_{j=1}^{p} b_{j}(t)<2,
$$

then the Green's function $G_{1}(t, s)$ of problem (1.1)-(1.3), (1.5) is nonpositive.

Proof. Let us substitute this $v(t)$, defined by (5.10), into the assertion of Theorem 5.1 .

$$
\begin{aligned}
-2 & +\sum_{i=1}^{p} a_{i}(t) \max _{i=1,2, \ldots, r}\left[v^{\prime}\left(t_{i}\right)-2 t_{i}\right] \\
& +\sum_{i=1}^{p} b_{i}(t) \max \left[\max _{i=1,2, \ldots, r} v\left(\frac{v^{\prime}\left(t_{i}\right)}{2}+t_{i}\right), \max _{i=0,1, \ldots, r} v\left(t_{i}\right)\right]<0,
\end{aligned}
$$

and we get the condition

$$
\Omega_{1} \sum_{j=1}^{p} a_{j}(t)+\Omega_{2} \sum_{j=1}^{p} b_{j}(t)<2 .
$$

Let us demonstrate this with two numeric examples.

Example 5.5. If $r=1, \gamma_{1}=\delta_{1}=1.2, t_{1}=\frac{1}{2}$ and $\omega=1$, then we get the following condition

$$
0.1 \sum_{j=1}^{p} a_{j}(t)+0.9 \sum_{j=1}^{p} b_{j}(t)<2
$$

for the nonpositivity of the Green's function.

Example 5.6. If $r=2, \gamma_{1}=\delta_{1}=\gamma_{2}=\delta_{2}=1.2, t_{1}=\frac{1}{3}, t_{2}=\frac{2}{3}$ and $\omega=1$, then we get the following condition

$$
0.933 \sum_{j=1}^{p} a_{j}(t)+1.114 \sum_{j=1}^{p} b_{j}(t)<2
$$

for the nonpositivity of the Green's function.

In the particular case $a_{j}(t)=0, j=1, \ldots, p$, we have the following corollary.

Corollary 5.7. If $b_{j} \geq 0,1 \leq \gamma_{k}, 1 \leq \delta_{k}, j=1, \ldots, r$, and

$$
\Omega_{2} \sum_{j=1}^{p} b_{j}(t)<2,
$$

then the Green's function $G_{1}(t, s)$ of problem (1.1)-(1.3), (1.5) is nonpositive.

Example 5.8. It is clear from the proof of Theorem 5.1 that our approach to study nonpositivity of the solution $x(t)$ for every nonnegative $f(t)$ can be extended to equation with general deviating argument (i.e. without the assumptions $\tau \geq 0, \theta \geq 0$ ). Consider the non-impulsive equation $x^{\prime \prime}(t)+x(1)=0$. The two-point boundary value problem for this equation with the conditions $x(0)=0, x^{\prime}(1)=0$ has the nontrivial solution $x(t)=t(2-t)$. This means that not for every $f(t)$ does there exist a solution for this problem and of course the Green's function does not exist. Computing $\Omega_{2}$ according to formula (5.13), we get $\Omega_{2}=v(1)=1$. We see 
that inequality (5.19) cannot be improved even in the non-impulsive case. For the equation $x^{\prime \prime}(t)+x(g(t))=f(t)$ with $g(t)$ "close" to 1, (5.19) will give "almost exact" estimate. The same could also be obtained in the case of the impulses $t_{i}$ situated "close" to 1 with $\gamma_{i}, \delta_{i}$ "close" to 1.

We can estimate the interval $[0, \omega]$, where the Green's function is nonpositive. More exactly, solving the initial value problem $(L v)(t) \leq 0, v(0)=0, v^{\prime}(0)=\mu>0$. If its solution $v(t)$ and its derivative $v^{\prime}(t)$ are positive, then the conditions of Theorem 5.1 are fulfilled and $G_{1}(t, s) \leq 0$.

Theorem 5.9. Assume that $a_{j} \leq 0, b_{j} \geq 0$ for $j=1, \ldots, p, 0<\gamma_{k} \leq 1,0<\delta_{k} \leq 1$ for $k=1, \ldots, r$ and there exists the function $v \in D$ and $\epsilon>0$ such that

$$
(L v)(t) \leq-\epsilon<0, \quad v(t)>0, \quad v^{\prime}(t)>0, \quad v^{\prime \prime}(t)<0, \quad t \in(0, \omega)
$$

where the differential operator $L$ is defined by (1.1). Then the Green's function $G_{2}(t, s)$ of (1.1)-(1.3), (1.6) satisfies the inequality $G_{2}(t, s) \leq 0,(t, s) \in[0, \omega] \times[0, \omega]$.

Proof. Let $v^{\prime \prime}(t)=z(t)$ where $z \in L_{\infty}$, then we can write

$$
v(t)=\int_{0}^{\omega} G_{0}^{2}(t, s) z(s) d s+V_{2}(t)
$$

where $G_{0}^{2}(t, s)$ is the Green's function of the problem (1.14), (1.2), (1.3), (1.6). After substitution we obtain

$$
z(t)=\left(K_{2} z\right)(t)+(L v)(t)-\sum_{j=1}^{p} a_{j}(t) V_{2}^{\prime}\left(t-\tau_{j}(t)\right)-\sum_{j=1}^{p} b_{j}(t) V_{2}\left(t-\theta_{j}(t)\right)
$$

where

$$
\begin{aligned}
\left(K_{2} z\right)(t)= & -\sum_{j=1}^{p} a_{j}(t) \int_{0}^{\omega} \frac{\partial}{\partial t} G_{0}^{2}\left(t-\tau_{j}(t), s\right) z(s) d s \\
& -\sum_{j=1}^{p} b_{j}(t) \int_{0}^{\omega} G_{0}^{2}\left(t-\theta_{j}(t), s\right) z(s) d s
\end{aligned}
$$

where $\frac{\partial}{\partial t} G_{0}^{2}\left(t-\tau_{j}(t), s\right)=0$ if $t-\tau_{j}(t)<0$ and $G_{0}^{2}\left(t-\theta_{j}(t), s\right)=0$ if $t-\theta_{j}(t)<0$. By Lemma 4.3, in the case $0<\gamma_{k} \leq 1,0<\delta_{k} \leq 1$ we have positivity of the operator $K_{2}: L_{\infty} \rightarrow L_{\infty}$. Now, from the condition about existence of $v$ satisfying (5.20), we get that $\tilde{z}(t)=v^{\prime \prime}(t)<0$, $z(t)=-\tilde{z}(t)>0$ and then there exists $\epsilon>0$ such that $z(t)-\left(K_{2} z\right)(t) \geq \epsilon$. From here, the spectral radius $\rho\left(K_{2}\right)$ of the operator $K_{2}: L_{\infty} \rightarrow L_{\infty}$ is less than one.

Assume now that $f \geq 0$ and demonstrate that $x \leq 0$. After the substitution

$$
x(t)=\int_{0}^{\omega} G_{0}^{2}(t, s) z(s) d s+V_{2}(t),
$$

we obtain

$$
z(t)=\left(K_{2} z\right)(t)+f(t)-\sum_{j=1}^{p} a_{j}(t) V_{2}^{\prime}\left(t-\tau_{j}(t)\right)-\sum_{j=1}^{p} b_{j}(t) V_{2}\left(t-\theta_{j}(t)\right),
$$


where $K_{2}$ is positive operator and its spectral radius $\rho\left(K_{2}\right)<1$. Then

$$
z(t)=\left(I-K_{2}\right)^{-1} f(t)=f(t)+K_{2} f(t)+K_{2}^{2} f(t)+\cdots
$$

is nonnegative, and $x$ obtained by (5.24) is nonpositive. Since this is true for every nonnegative function $f$, we can make a conclusion that the solution of problem (1.1)-(1.3), (1.6) exists for every summable function $f$. Now it is clear that the solution of this problem has the integral representation (see (1.20)) with the kernel $G_{2}(t, s)$ (Green's function of (1.1)-(1.3), (1.6)). We proved that, for every nonpositive right hand side function $f$, the solution $x$ is nonpositive. From here, it follows that $G_{2}(t, s) \leq 0$.

Theorem 5.9 has been proven.

Example 5.10. Let us now find an example of a function $v$ satisfying all conditions of Theorem 5.9. To this end, let us start with $v(t)=(\omega+t)(\omega-t)$ in the interval $t \in\left[0, t_{1}\right)$ where $\epsilon$ is a small positive constant. The function $v$ in the rest of the intervals will be of the form

$$
v(t)=v\left(t_{i}\right)+v^{\prime}\left(t_{i}\right)\left(t-t_{i}\right)-\left(t-t_{i}\right)^{2}, \quad t \in\left[t_{i}, t_{i+1}\right), \quad i=1, \ldots, r, \quad t_{r+1}=\omega
$$

where

$$
\left\{\begin{array}{l}
v\left(t_{i}\right)=\gamma_{i} v\left(t_{i}-0\right), \\
v^{\prime}\left(t_{i}\right)=\delta_{i} v^{\prime}\left(t_{i}-0\right) .
\end{array}\right.
$$

Thus

$$
\left\{\begin{array}{l}
v(t)=(\omega+t)(\omega-t), \quad t \in\left[0, t_{1}\right), \\
v(t)=v\left(t_{i}\right)+v^{\prime}\left(t_{i}\right)\left(t-t_{i}\right)-\left(t-t_{i}\right)^{2}, \quad t \in\left[t_{i}, t_{i+1}\right),
\end{array}\right.
$$

where $v\left(t_{i}\right)$ and $v^{\prime}\left(t_{i}\right)$ can be represented in the form

$$
\left\{\begin{aligned}
v\left(t_{i}\right)= & \left(\omega+t_{1}\right)\left(\omega-t_{1}\right) \prod_{j=1}^{i} \gamma_{j}+\sum_{k=2}^{i} v^{\prime}\left(t_{k}\right)\left(t_{k}-t_{k-1}\right) \prod_{j=k}^{i} \gamma_{j} \\
& -\sum_{k=2}^{i}\left(t_{k}-t_{k-1}\right)^{2} \prod_{j=k}^{i} \gamma_{j} \\
v^{\prime}\left(t_{i}\right)= & -2 t_{1} \prod_{j=1}^{i} \delta_{j}-2 \sum_{k=2}^{i}\left(t_{k}-t_{k-1}\right) \prod_{j=k}^{i} \delta_{j} .
\end{aligned}\right.
$$

Let us assume that $v(t)>0$ and substitute this $v(t)$ into the assertion of Theorem 5.9

For the next corollary, we use the following notation:

$$
\begin{aligned}
& \Omega_{1}=\max _{i=1,2, \ldots, r}\left|v^{\prime}\left(t_{i}\right)-2 t_{i}\right|, \\
& \Omega_{2}=\max \left[\max _{i=1,2, \ldots, r} v\left(\frac{v^{\prime}\left(t_{i}\right)}{2}+t_{i}\right), \max _{i=0,1, \ldots, r} v\left(t_{i}\right)\right],
\end{aligned}
$$

where $v\left(t_{r+1}\right)=v(\omega)$.

Corollary 5.11. If $a_{j} \leq 0, b_{j} \geq 0,0<\gamma_{k} \leq 1,0<\delta_{k} \leq 1, j=1, \ldots, p, v(t)$ defined by (5.29) is positive for $t \in(0, \omega)$ and

$$
\Omega_{1} \sum_{j=1}^{p}\left|a_{j}(t)\right|+\Omega_{2} \sum_{j=1}^{p} b_{j}(t)<2,
$$

then the Green's function $G_{2}(t, s)$ of problem (1.1)-(1.3), (1.6) is nonpositive. 
Proof. Let us substitute this $v(t)$, defined by (5.29), into the assertion of Theorem 5.9

$$
\begin{aligned}
-2 & +\sum_{i=1}^{p}\left|a_{i}(t)\right| \max _{i=1,2, \ldots, r}\left|v^{\prime}\left(t_{i}\right)-2 t_{i}\right| \\
& +\sum_{i=1}^{p} b_{i}(t) \max \left[\max _{i=1,2, \ldots, r} v\left(\frac{v^{\prime}\left(t_{i}\right)}{2}+t_{i}\right), \max _{i=0,1, \ldots, r} v\left(t_{i}\right)\right]<0,
\end{aligned}
$$

and we get the condition (5.33).

Let us demonstrate this with two numeric examples.

Example 5.12. If $r=1, \gamma_{1}=\delta_{1}=1.2, t_{1}=\frac{1}{2}$ and $\omega=1$, then $\Omega_{1}=2.2, \Omega_{2}=1$ and we get the following condition

$$
2.2 \sum_{j=1}^{p} a_{j}(t)+1 \sum_{j=1}^{p} b_{j}(t)<2
$$

for the nonpositivity of the Green's function.

Example 5.13. If $r=2, \gamma_{1}=\delta_{1}=\gamma_{2}=\delta_{2}=1.2, t_{1}=\frac{1}{3}, t_{2}=\frac{2}{3}$ and $\omega=1$, then $\Omega_{1}=3.888, \Omega_{2}=\frac{16}{15}$ and we get the following condition

$$
3.888 \sum_{j=1}^{p} a_{j}(t)+\frac{16}{15} \sum_{j=1}^{p} b_{j}(t)<2
$$

for the nonpositivity of the Green's function.

In the particular case $a_{j}(t)=0, j=1, \ldots, p$, we have the following result.

Corollary 5.14. If $b_{j} \geq 0,0<\gamma_{k} \leq 1,0<\delta_{k} \leq 1, j=1, \ldots, r$, and

$$
\Omega_{2} \sum_{j=1}^{p} b_{j}(t)<2
$$

then the Green's function $G_{2}(t, s)$ of problem (1.1)-(1.3), (1.6) is nonpositive.

Remark 5.15. In the case of non-impulsive equation (1.1), where $a_{j}=0, b_{j} \geq 0$ for $j=1, \ldots, p$, we have $\Omega_{2}=\max _{i=0, \ldots, r+1} v\left(t_{i}\right)=v(0)=\omega^{2}$ and condition (5.33) will be of the form

$$
\sum_{j=1}^{p} b_{j}(t)<\frac{2}{\omega^{2}}
$$

This condition cannot be improved. Actually, for the equation $x^{\prime \prime}(t)+x(0)=f(t), t \in$ $[0, \omega]$, condition (5.33) is exact one, since the function $x(t)=(\omega-t)(t+\omega)$ is a nontrivial solution of the problem $x^{\prime \prime}(t)+x(0)=0, t \in[0, \omega], x^{\prime}(0)=0, x(\omega)=0$.

It is clear that our condition will be close to optimal also for $x^{\prime \prime}(t)+x(g(t))=f(t)$, where $g(t)$ is small enough. 


\section{References}

[1] R. P. Agarwal, L. Berezansky, E. Braverman, A. Domoshnitsky, Nonoscillation theory of functional differential equations with applications, Springer, 2012. MR2908263; url

[2] N. V. Azbelev, V. P. Maksimov, L. F. Rakhmatullina, Introduction to the theory of functional differential equations, Advanced Series in Math. Science and Engineering, Vol. 3, World Federation Publisher Company, Atlanta, GA, 1995. MR1422013

[3] D. Bainov, Y. DomshlaK, P. Simeonov, Sturmian comparison theory for impulsive differential inequalities and equations, Arch. Math. (Basel) 67(1996), 35-49. MR1392051; url

[4] D. Bainov, P. Simeonov, Impulsive differential equations, Pitman Monogpaphs and Surveys in Pure and Applied Mathematics, Vol. 66, Longman Scientific, Harlow, 1993. MR1266625

[5] L. Berezansky, E. Braverman, Oscillation of a linear delay impulsive differential equation, Commun. Appl. Nonlinear Anal. 3(1996), 61-77. MR1365179

[6] L. Berezansky, E. Braverman, Oscillation and other properties of linear impulsive and nonimpulsive delay equations, Appl. Math. Lett. 16(2003), 1025-1030. MR2013068; url

[7] Y.-Sh. Chen, YANG-ShaO, W-Zh. Feng, Oscillation of second order nonlinear ODE with impulses, J. Math. Anal. Appl. 210(1997), 150-169. MR1449514; url

[8] A. Domoshnitsky, M. Drakhlin, On boundary value problems for first order impulse functional differential equations, in: J. Henderson (Ed.), Boundary value problems for functional-differential equations, World Scientific, River Edge, NJ, 1995, pp. 107-117. MR1375468; url

[9] A. Domoshnitsky, M. Drakhlin, Nonoscillation of first order impulse differential equations with delay, J. Math. Anal. Appl. 206(1997), 254-269. MR1429290; url

[10] A. Domoshnitsky, M. Drakhlin, E. Litsyn, Nth order functional-differential equations with impulses, Adv. Math. Sci. Appl. 8(1998), No. 2, 987-996. MR1657212

[11] A. Domoshnitsky, G. Landsman, S. Yanetz, About sign-constancy of Green's functions for impulsive second order delay equations, Opuscula Math. 34(2014), No. 2, 339-362. MR3200260; url

[12] A. Domoshnitsky, I. Volinsky, R. ShkLyar, About Green's functions for impulsive differential equations, Funct. Differ. Equ. 20(2013), No. 1-2, 55-81. MR3328886

[13] M. FENG, D. XIE, Multiple positive solutions of multi-point boundary value problem for second-order impulsive differential equations, J. Comput. Appl. Math. 223(2009), 438-448. MR2463127; url

[14] L. P. Gimenes, M. Federson, Existence and impulsive stability for second order retarded differential equations, Appl. Math. Comput. 177(2006), No. 1, 44-62. MR2234496; url

[15] S. Hu, V. Lakshmikantham, Periodic boundary value problems for second order impulsive differential systems, Nonlinear Anal. 13(1989), No. 1, 75-85. MR973370; url 
[16] J. JiAnG, L. LiU, Y. WU, Positive solutions for second order impulsive differential equations with Stieltjes integral boundary conditions, Adv. Difference Equ. 2012, 2012:124, 18 pp. MR3016047; url

[17] M. A. Krasnoselskit, G. M. VainikKo, P. P. Zabreiko, Ja. B. Rutitskit, V. Ja. Stezenko, Approximate methods for solving operator equations (in Russian), Moscow, Nauka, 1969. MR0259635

[18] V. Lakshmikantham, D. D. Bainov, P. S. Simeonov, Theory of impulsive differential equations, World Scientific, Singapore, 1989. MR1082551; url

[19] J. Li, J. J. Nieto, J. Shen, Impulsive periodic boundary value problems of first-order differential equations, J. Math. Anal. Appl. 325(2007), 226-236. MR2273040; url

[20] X. LI, P. WeNG, Impulsive stabilization of two kinds of second-order linear delay differential equations, J. Math. Anal. Appl. 291(2004), 270-281. MR2034073; url

[21] X. Lin, D. JIANG, Multiple positive solutions of Dirichlet boundary value problems for second order impulsive differential equations, J. Math. Anal. Appl. 321(2006), 501-514. MR2241134; url

[22] S. G. Pandit, S. G. Deo, Differential systems involving impulses, Lecture Notes in Mathematics, Vol. 954, Springer-Verlag, Berlin, 1982. MR674119

[23] A. M. Samoilenko, A. N. Perestyuk, Impulsive differential equations, World Scientific, Singapore, 1995. MR1355787; url

[24] Y. L. TIAN, P. X. Weng, J. J. YANG, Nonoscillation for a second order linear delay differential equation with impulses, Acta Math. Appl. Sin. Engl. Ser. 20(2004), 101-114. MR2052709; url

[25] J. YAN, A. ZhAO, Oscillation and stability of linear impulsive delay differential equations, J. Math. Anal. Appl. 227(1998), 187-194. MR1652915; url

[26] S. G. Zavalishchin, A. N. Sesekin, Dynamic impulse systems. Theory and applications, Mathematics and its Applications, Vol. 394, Kluwer Academic Publishers Group, Dordrecht, 1997. MR1441079; url 